\section{PTU-114 EXPRESSION OF TOLL-LIKE RECEPTOR (TLR)-2 AND -4 IN THE INTESTINAL CRYPT EPITHELIAL CELLS IN INFLAMMATORY BOWEL DISEASE (IBD)}

\section{doi:10.1136/gutjnl-2012-302514c.114}

${ }^{1,2} \mathrm{M} \mathrm{R}$ L Brown, ${ }^{*} \mathrm{~A}$ Robins, ${ }^{1} \mathrm{Y} \mathrm{R}$ Mahida. ${ }^{1}$ Institute of Infection, Immunity $\&$ Inflammation, University of Nottingham, Nottingham, UK; ${ }^{2}$ NIHR Biomedical Research Unit in the Nottingham Digestive Diseases Centre, Nottingham University Hospitals NHS Trust, Nottingham, UK

Introduction TLRs are pattern recognition receptors which detect conserved molecular patterns on commensal and pathogenic bacteria. Studies in mice have shown that TLR signalling protects against the development of colitis but may exacerbate established colitis. Epithelial stem cells and their progeny are located in crypts of the small and large intestine. We have investigated TLR2 and TLR4 expression in ulcerative colitis (UC) and Crohn's disease (CD) crypt intestinal epithelial cells (IEC).

Methods Ileal and colonic mucosal samples were obtained from operation resection specimens and were histologically normal ( $>5 \mathrm{~cm}$ from cancer; normal controls) or inflamed (CD or UC). Additionally, paired samples from histologically normal (proximal colon) and inflamed (distal colon) mucosa were obtained from five colectomy specimens with isolated left-sided UC. Crypt IECs were isolated using EDTA and pancreatin. Expression of mRNA transcripts was studied by conventional and real-time RT-PCR. Cell surface TLR protein was studied by flow cytometry (expressed as median fluorescent intensity, MFI). TLR expression was also studied by immunofluorescent (IF) staining of tissue sections. Data are expressed as median (IOR).

Results Relative to normal controls, TLR2 mRNA expression in IEC was significantly elevated in inflamed colonic UC [3.18-fold $(1.03-10.40), \mathrm{p}=0.003]$ and inflamed colonic CD [3.45 (0.80-5.40), $\mathrm{p}=0.012]$. TLR4 mRNA was significantly elevated in normal colonic UC $[1.90$ (1.69-4.31], $\mathrm{p}=0.017)$, inflamed colonic UC [2.33 (1.15-4.45), $\mathrm{p}=0.024]$, inflamed colonic CD [1.71 (1.00-4.32), $p=0.042]$ and inflamed ileal CD [1.84 (1.43-4.66), $p=0.03]$. In paired analyses, IEC TLR2 and TLR4 mRNA expression was not significantly different in normal UC mucosa compared to inflamed UC mucosa. Compared to IEC from normal control colon, IEC from colonic CD showed significantly greater expression of cell surface TLR2 protein [MFI $10.1(2.4-29.0)$ vs 33.1 (17.7-107.5), $\mathrm{p}=0.05$ ] and TLR4 protein [MFI 12.1 (4.9-25.3) vs 40.9 (23.2-103.5), $\mathrm{p}=0.04]$. IF staining confirmed TLR 2 and TLR 4 protein expression on the luminal surface of crypt IEC.

Conclusion IEC expression of TLR4 mRNA was up-regulated in inflamed and un-inflamed colonic and ileal IBD. TLR2 mRNA was up-regulated in inflamed colonic IBD. Interestingly, TLR2 and TLR4 mRNA expression was similar in IECs isolated from un-inflamed, histologically normal mucosa as it was in IECs isolated from inflamed mucosa in UC. This may suggest TLR2 and TLR4 upregulation is a primary rather than secondary event in UC. Moreover, TLR2 and TLR4 protein was expressed in greater amounts on the surface of IECs in CD.

Competing interests None declared.

\section{PTU-115 EFFICACY OF MYCOPHENOLATE MOFETIL THERAPY IN THE MANAGEMENT OF INFLAMMATORY BOWEL DISEASE}

doi:10.1136/gutjnl-2012-302514c.115

M R Smith, ${ }^{*}$ L Wood, J Slater, S C Cooper. Gastroenterology Department, Russells Hall Hospital, Dudley Group NHS Foundation Trust, Dudley, UK

Introduction The role of Mycophenolate mofetil (MMF) as an immunomodulatory drug in managing inflammatory bowel disease is yet to be fully defined. We reviewed our experience of the efficacy, safety and tolerability of MMF in treating patients with refractory inflammatory bowel disease.

Methods A retrospective analysis was performed of the case records of all patients treated with MMF for inflammatory bowel disease at our institution between 2003 and 2011. Remission was assessed by reviewing clinical, endoscopic and laboratory indices.

Results We identified 36 patients, 23 male (64\%) with a median age 46 yrs (range 19-75). Disease was classified as Crohn's disease in 19, ulcerative colitis 16 , indeterminate colitis. ${ }^{1} 33$ patients $(92 \%)$ had previously received azathioprine; 32 discontinued this due to sideeffects. Five patients had undergone surgery for small bowel Crohn's disease. The starting dose of MMF was between $500 \mathrm{mg}$ and $2 \mathrm{~g}$ daily, titrated to a dose of $2 \mathrm{~g}$ daily as tolerated. 26 patients $(72 \%)$ were concurrently taking oral corticosteroids, and $18(50 \%)$ were taking an oral 5-aminosalicylate. At 8 weeks, 29 patients (81\%) had either achieved acute remission or maintained previous remission. Drug side-effects were experienced by eight patients (22\%)-these symptoms were managed successfully by dose reduction in six patients $(75 \%)$, with discontinuation in two patients. There were no serious haematological or other adverse drug effects. After 6 months of treatment, 33 patients continued to take MMF of which 19 patients (58\%) had achieved sustained steroid-free remission. Median length of MMF treatment observed was 21.5 months (IOR 9.7-31.6). At the end of the observation period, 29 patients (81\%) remained on MMF. 13 patients (36\% of the original treatment group; UC 7, Crohn's 5) remained in steroid-free remission with median time of remission 21.4 months (IOR 11.0-30.0). A further 13 patients achieved sustained remission with the addition of corticosteroids and/or anti-TNF therapy. Five patients (14\% overall) were refractory to all medical therapy and underwent surgery: colectomy for UC 3, right hemicolectomy for Crohn's. ${ }^{2}$

Conclusion From our experience, MMF may represent a promising alternative treatment for inducing and maintaining remission in patients intolerant of or unable to receive thiopurines. It appears well tolerated with a good safety profile in thiopurine intolerant subjects.

Competing interests None declared.

\section{PTU-116 EXPRESSION OF NLRP3 INFLAMMASOME PROTEINS IN NORMAL AND CROHN'S DISEASE COLON}

doi:10.1136/gutjnl-2012-302514c.116

${ }^{1} \mathrm{M}$ B Sprakes, ${ }^{*}{ }^{2} \mathrm{M}$ McDermott, ${ }^{1} \mathrm{~J}$ Hamlin. ${ }^{1}$ Gastroenterology, Leeds Teaching Hospitals, Leeds, UK; ${ }^{2}$ Experimental Rheumatology, Leeds Institute of Molecular Medicine, Leeds, UK

Introduction The pathogen recognition receptor, NLRP3, and its inflammasome are implicated in the innate immunity. ${ }^{1}$ Both intracellular and extracellular stressors induce its activation including the bacterial protein muramyl dipeptide, ${ }^{2}$ also recognised by NLRC2 (NOD2) which is known to be associated with Crohn's disease (CD). Little is known about expression of the NLRP3 inflammasome in normal gastrointestinal tract (GIT) or in CD, therefore the expression profile of four inflammasome proteins in the GIT has been investigated in this study.

Methods Paraffin embedded colonic tissue from healthy controls (HC) and CD patients were cut and fixed on slides. Protein antigens were exposed by pressure cooker heating in $0.01 \%$ antigen retrieval solution and water. Following application of a primary antibody (anti-NLRP3, anti-ASC, anti-pyrin, anti-caspase-1) the proteins were observed by washing in $\mathrm{DAB} /$ chromogen solution, following incubation with a Horseradish peroxidase-labelled secondary antibody. Degree of staining was assessed using a validated Quick score model $^{3}$ where intensity of staining is scored $0-3$, and proportion of 
cells stained is scored 0-5, a maximum Quick score per sample of 8. Differences in expression between $\mathrm{HC}$ and $\mathrm{CD}$ samples were analysed using Mann-Whitney U test.

Results Colonic biopsies from $20 \mathrm{HC}$ and $20 \mathrm{CD}$ patients were stained for NLRP3, ASC, Pyrin and Caspase-1 protein expression. All four proteins were expressed in $\mathrm{HC}$ and $\mathrm{CD}$ tissue. There were significant differences in expression of all 4 proteins when $\mathrm{HC}$ and colonic CD samples were compared (Abstract PTU-116 table 1).

Abstract PTU-116 Table 1 Difference in expression of inflammasome proteins

\begin{tabular}{lclc}
\hline Protein & $\begin{array}{l}\text { Quick score } \\
\text { HC median (IOR) }\end{array}$ & $\begin{array}{l}\text { Quick score } \\
\text { CD median (IOR) }\end{array}$ & p Value \\
\hline NLRP3 & $2(2-5)$ & $7(6-7.5)$ & $<0.001$ \\
ASC & $5.5(4-7)$ & $7(6-8)$ & 0.02 \\
Pyrin & $6(5-6.25)$ & $7(6-8)$ & 0.05 \\
Caspase-1 & $4(3-5)$ & $5(4-6)$ & 0.02 \\
\hline
\end{tabular}

Conclusion The NLRP3 inflammasome is expressed in normal, healthy GIT mucosa and is upregulated in colonic tissue from CD patients with active disease. This suggests the NLRP3 inflammasome may play a role in mucosal immunity in CD patients.

Competing interests None declared.

\section{REFERENCES}

1. Petrilli V, Dostert C, Muruve DA, et al. The inflammasome: a danger complex triggering innate immunity. Curr Opin Immunol 2007;19:615-22.

2. Martinon F, Agostini L, Meylan E. Identification of bacterial muramyl dipeptide as activator of the NALP3/cryopyrin inflammasome. Curr Biol 2004;14:1929-34.

3. Detre S, Saclani Jotti G, Dowsett M. A "quickscore" method for immunohistochemical semiquantification: validation for oestrogen receptor in breast carcinomas. J Clin Path 1995;48:876-8.

\section{PTU-117 ANALYSIS OF THE INCIDENTAL DIAGNOSIS OF INFLAMMATORY BOWEL DISEASE MADE DURING THE SCOTTISH BOWEL CANCER SCREENING PROGRAMME}

doi:10.1136/gutjnl-2012-302514c.117

${ }^{1} \mathrm{M}$ A Zahid, ${ }^{1} \mathrm{C}$ Noble, ${ }^{1} \mathrm{C}$ W Lees, ${ }^{1} \mathrm{~J}$ Satsangi, ${ }^{2} \mathrm{G}$ Wilson, ${ }^{3} \mathrm{~A}$ Lessells. ${ }^{1}$ Gastroenterology, Western General Hospital, Edinburgh, UK; ${ }^{2}$ Colorectal Surgery, Western General Hospital, Edinburgh, UK; ${ }^{3}$ Pathology, Western General Hospital, Edinburgh, UK

Introduction The Scottish Bowel Screening Programme (SBSP) has been running in Scotland since March 2008 in patients aged between 50 and 74 . The aim of this study was to quantify the number of new cases of inflammatory bowel disease (IBD) diagnosed as part of the SBSP in South East Scotland. The progression of these patients was also assessed.

Methods All the patients who had a bowel screening colonosocpy during the first 3 years of SBSP in South East Scotland were identified (screening population 800000 ). Histologically confirmed cases of IBD were isolated and information like symptoms at the time of diagnosis, risk factors and initial treatment was collated. The severity of illness was retrospectively assessed using Harvey Bradshaw index and Mayo score for Crohn's disease (CD) and ulcerative colitis (UC) respectively. The patients' progress following diagnosis was also assessed.

Results $51(1.4 \%)$ patients with IBD were diagnosed out of total 3655 procedures performed between June 2008 and April 2011. Of these, $12(0.3 \%)$ patients had previous diagnosis of IBD and were excluded from study. In patients with a new diagnosis of IBD $(n=39)$, significantly more males $30(77 \%)$, with a mean age of 63 at diagnosis, were diagnosed with IBD than females (9-23\%), $(p<0.001)$, mean age of $67.12(30 \%)$ Patients were diagnosed with
CD, 16 (41\%) had UC and 11 (28.2\%) had IBD unclassified (IBDU) The disease location is shown in Abstract PTU-117 table 1. 26 (67\%) patients were symptomatic at the time of diagnosis with a mean Mayo score of 2.4 for UC and a mean Harvey Bradshaw score of 1.4 for $\mathrm{CD}$ group. 34 (87\%) of patients were in remission in the followup period of 6 to 30 months. 9 (23\%) had no treatment, $19(48.4 \%)$ had oral or topical mesalazine, $4(10 \%)$ had oral steroids while 3 $(7.6 \%)$ patients required both oral steroids and mesalazine. Five patients were unresponsive to initial therapy (2-CD, 1-UC, 2-IBDU). Among these, three patients required Azathioprine, two had steroids and one required methotrexate after developing inflammatory arthritis.

Conclusion In this cohort of 3655 patients, IBD was diagnosed in $1.1 \%$ of patients. This is in line with published data. ${ }^{1}$ There was a preponderance of male patients. When assessed the majority of patients had previous symptoms and following diagnosis their IBD followed a benign course.

Abstract PTU-117 Table 1 Site of involvement on colonoscopy

\begin{tabular}{llllll}
\hline Diagnosis & $\begin{array}{l}\text { Rectum } \\
(\mathbf{n}=\mathbf{1 1})\end{array}$ & $\begin{array}{l}\text { Rectosigmoid } \\
(\mathbf{n}=\mathbf{1 4})\end{array}$ & $\begin{array}{l}\text { Left colon } \\
(\mathbf{n}=\mathbf{2})\end{array}$ & $\begin{array}{l}\text { Pancolon } \\
(\mathbf{n}=\mathbf{8})\end{array}$ & $\begin{array}{l}\text { Ileum } \\
(\mathbf{n}=\mathbf{4})\end{array}$ \\
\hline UC & $9(23 \%)$ & $3(7.6 \%)$ & $1(2.5 \%)$ & $3(7.6 \%)$ & \\
CD & 0 & $5(12.8 \%)$ & 0 & $3(7.6 \%)$ & $4(10.2 \%)$ \\
IBDU & $2(5 \%)$ & $6(15.3 \%)$ & $1(2.5 \%)$ & $2(5 \%)$ & 0 \\
\hline
\end{tabular}

Competing interests None declared.

\section{REFERENCE}

1. Louis-Auguste $\mathbf{J}$, Cohen $P$, Martin J, et al. New diagnoses of inflammatory bowel disease during bowel cancer screening colonoscopy. Gut 2011;60:A217.

\section{PTU-118 A DIAGNOSTIC ACCURACY META-ANALYSIS OF ENDOANAL ULTRASOUND AND MRI FOR PERIANAL FISTULA ASSESSMENT}

doi:10.1136/gutjnl-2012-302514c.118

${ }^{1} \mathrm{M}$ R S Siddiqui, ${ }^{*} \mathrm{H}$ Ashrafian, ${ }^{1} \mathrm{P}$ Tozer, ${ }^{1} \mathrm{~N}$ Daulatzai, ${ }^{3} \mathrm{D}$ Burling, ${ }^{3} \mathrm{~A}$ Hart,

${ }^{2} \mathrm{~T}$ Athanasiou, ${ }^{1} \mathrm{R}$ K Phillips. ${ }^{1}$ Colorectal Surgery, St Marks Hospital, London, UK; ${ }^{2}$ Imperial College, London, UK; ${ }^{3}$ St Marks Hospital, London, UK

Introduction Imaging modalities such as endoanal ultrasound or MRI can be useful preoperative adjuncts prior to the appropriate surgical intervention for perianal fistulas. We present a systematic review of published literature comparing endoanal ultrasound with MRI for the assessment of idiopathic and Crohn's perianal fistulas.

Methods A meta-analysis was performed to obtain pooled values for specificity and sensitivity. Electronic databases were searched from January 1970 to October 2010 for published studies.

Results Four studies were used in our analysis. There were 241 fistulas in the ultrasound group and 240 in the magnetic resonance group. The combined sensitivity and specificity of magnetic resonance for fistula detection were 0.87 (95\% CI 0.63 to 0.96 ) and 0.69 (95\% CI 0.51 to 0.82 ). There was a high degree of heterogeneity between studies reporting on MRI sensitivity ( $\left.\mathrm{df}=3, \mathrm{I}^{2}=93 \%\right)$. This compares to a sensitivity and specificity for endoanal ultrasound of 0.87 (95\% CI 0.70 to 0.95 ) and 0.43 (95\% CI 0.21 to 0.69 ) respectively. There was a high degree of heterogeneity between studies reporting on EAUS sensitivity ( $\mathrm{df}=3, \mathrm{I}^{2}=92 \%$ ).

Conclusion From the available literature, the summarised performance characteristics for MRI and EAUS demonstrate comparable sensitivities at detecting perianal fistulas, although the specificity for MRI was higher than that for EAUS. Both specificity values are however considered to be diagnostically poor. The high degree of 